\title{
El campo organizacional de la agricultura en el valle del Yaqui en Sonora
}

\section{The Organizational Field of Agriculture in the Yaqui Valley in Sonora}

\author{
José Carlos López Figueroa* (D) https://orcid.org/0000-0001-9840-6241 \\ Sergio Ochoa Jiménez ${ }^{* *}$ (D) https://orcid.org/0000-0003-1848-3760 \\ José Guadalupe Flores López ${ }^{\star \star \star}$ (iD https://orcid.org/0000-0002-6380-5135
}

\section{Resumen}

Objetivo: explicar el proceso de estructuración del valle del Yaqui como campo organizacional. Metodología: se realizaron I3 entrevistas, complementadas con notas de campo, instrumentos que recogieron el testimonio de siete organizaciones y el de un asesor jurídico que permitieron construir cuatro mapas analíticos: el de los activos físico-territoriales, el de los actores, el de los conflictos y el de las redes de actores. Resultados: se visualiza el valle como un campo organizacional con el énfasis puesto en los actores agrupados en seis niveles para dar respuesta a las cuatro etapas que DiMaggio y Powell proponen para la estructuración de un campo organizacional: I) la interacción, 2) las estructuras interorganizacionales, 3) el incremento en la carga de información y 4) el desarrollo de una conciencia mutua. Limitaciones: debido a la diversidad de los actores integrantes del territorio, no ha sido posible considerar a todo el universo y sus posibles relaciones, pero se incluye a la mayoría. Valor: se propone el estudio del territorio como un campo organizacional bajo la perspectiva de los estudios organizacionales. Conclusiones: los estudios organizacionales y sus líneas de investigación son una alternativa para potenciar el análisis y la comprensión del territorio.

Palabras clave: estudios organizacionales; campo organizacional; territorio; organizaciones agrícolas; valle del Yaqui.

Cómo citar: López Figueroa, J. C., Ochoa Jiménez, S., y Flores López, J. G. (2022). El campo organizacional de la agricultura en el valle del Yaqui en Sonora. región y sociedad, 34, e I519. doi: I0.22198/rys2022/34/I5I9

* Instituto Tecnológico de Sonora, Departamento de Ciencias Administrativas, Unidad Obregón, Campus Centro. 5 de Febrero, núm. 818 Sur, C.P. 85000, Ciudad Obregón, Sonora, México. Correo electrónico: j.lopezf11@gmail.com

${ }^{\star *}$ Autor para correspondencia. Instituto Tecnológico de Sonora, Departamento de Ciencias Administrativas, Unidad Obregón, Campus Centro. 5 de Febrero, núm. 818 Sur, C.P. 85000, Ciudad Obregón, Sonora, México. Correo electrónico: sochoa@itson.edu.mx

${ }^{* * *}$ Instituto Tecnológico de Sonora, Departamento de Ciencias Administrativas, Unidad Obregón, Campus Centro. 5 de Febrero, núm. 818 Sur, C.P. 85000, Ciudad Obregón, Sonora, México. Correo electrónico: jose.flores@itson.edu.mx

Recibido: 13 de julio de 2021

Aceptado: 15 de diciembre de 2021

Liberado: 10 de febrero de 2022 


\section{Introducción}

Teoría institucional ( $\mathrm{TI})$ es un término que cubre un amplio corpus de literatura que centra su análisis en la importancia de la institución (Hoffman y Wooten, 2008), y es posible encontrar diversidad de enfoques para estudiar el institucionalismo (Vargas-Hernández, 2008), los cuales se aglomeran en varias racionalidades teórico-metodológicas y se anclan en algunas disciplinas, como la administración, la teoría de la organización y los estudios organizacionales (EO) (Montaño, 2020). De acuerdo con DiMaggio y Powell (1999) y Hoffman y Wooten (2008), el concepto central del nuevo institucionalismo (NI) ha sido el de campo organizacional, término que emerge como un nuevo nivel de análisis para la comprensión de los procesos institucionales (Reay y Hinings, 2005; Scott, 2012a).

El presente artículo recurre al estudio de casos para describir la estructuración de un campo organizacional (la agricultura del valle del Yaqui) y se basa en los argumentos que desarrolla la teoría del NI. Este territorio puede verse como un campo organizacional ya estructurado, puesto que ha pasado por diversos hechos históricos que han marcado su estructura agraria.

Desde una perspectiva documental, pueden mencionarse algunos momentos importantes: la expropiación de terrenos durante la gestión del presidente Lázaro Cárdenas (1934-1940) que dio origen a la institución de los ejidos colectivos; el reparto agrario en 1976 durante la presidencia de Luis Echeverría (1970-1976), que afectó más o menos 37000 hectáreas, para incrementar la cantidad de ejidos colectivos; y la reforma al artículo 27 constitucional con Carlos Salinas de Gortari (1988-1994) en 1992, que puso fin al reparto agrario y otorgó dominio pleno a los ejidatarios sobre sus parcelas.

Teniendo en cuenta el contexto anterior, se ha decidido tomar como punto de partida la existencia del campo agrícola a partir de la presencia de actores relevantes, sus relaciones y los intercambios de información entre ellos. Para alcanzar el objetivo planteado, el artículo se divide en cinco apartados: el primero aborda como tema central la posible vinculación entre el concepto de territorio y los EO. Dicho concepto se asocia con varias disciplinas de las ciencias sociales, como la geografía, la economía, la sociología y la antropología, entre otras, mientras que los EO tienen una naturaleza multidisciplinaria pues presentan una interacción con las ciencias humanas y sociales. En el primer apartado se abordan diferentes conceptos y dimensiones del territorio, así como distintas perspectivas del campo organizacional, entre las que sobresalen la visión estructural, la cognitiva y una en relación con el poder. La vinculación entre el territorio y los EO deriva de la perspectiva social del primer término en cuanto que espacio de diálogo entre actores, por lo que esta visión permite realizar el vínculo con la noción de campo organizacional al identificar a todos los actores importantes y sus relaciones.

El segundo apartado ilustra la diversidad de formas en las que el valle del Yaqui ha sido estudiado. En él se diagnostica el territorio para señalar algunas de sus características distintivas y se abordan varios estudios en relación con las dimensiones territoriales: la social, la económica, la política y la cultural. 
Teniendo presente el contexto teórico de los primeros dos apartados, el tercero versa sobre las consideraciones metodológicas. En éste se describen las técnicas de recolección de datos: la entrevista semiestructurada, las notas de campo y la construcción de mapas para analizar la información relacionada con las cuatro etapas que permiten estructurar los campos. También se da cuenta de la participación de las siete organizaciones productoras, entre las que se destacan la de los ejidatarios, la de los comuneros, la de los integrantes de una sociedad de producción rural y la de los arrendatarios, es decir, aquellos que pagan por usufructuar la tierra.

El cuarto apartado presenta en dos secciones los resultados de la investigación. La primera trata sobre la idea de cambio, identifica a los actores integrantes del campo organizacional y los ubica en tres etapas: de 1930 a 1970; de 1971 a 1992 (periodo anterior a la reforma al artículo 27 constitucional); y después de la reforma al artículo 27 . Al final de esa sección, se propone una tipología de seis tipos de actores organizacionales. En la segunda sección, a partir de las cuatro etapas que sugieren DiMaggio y Powell (1999) -la interacción, las estructuras interorganizacionales de dominio, un incremento en la carga de información y el desarrollo de una conciencia entre los actores-, se describe de forma detallada la estructuración del campo organizacional. El último apartado está dedicado a las conclusiones, en las que se reconoce la complejidad territorial desde los EO y se revisan las instituciones, las relaciones de poder, las lógicas institucionales y el isomorfismo, entre otros temas. No obstante, se centran más en la proposición del concepto de campo organizacional como unidad de análisis para la intervención de los territorios desde la perspectiva de la gobernanza, que resulta ser una alternativa para el diseño de políticas públicas locales.

\section{Pensar el territorio desde los estudios organizacionales}

El concepto de territorio tiene una larga existencia. Como señala Capel (2016), “la palabra 'territorio' procede del latín territorium, y se encuentra en castellano, al igual que en francés, por lo menos desde el siglo XIII” (p. 3). Este concepto mantiene su importancia hasta finales del siglo XIX (Llanos-Hernández, 2010), cuando el geógrafo Paul Vidal de La Blache inicia los trabajos que tenían la noción de región como contrapunto de la noción de territorio (Schneider y Peyré, 2006). Años más tarde, en la década de 1970, el concepto de territorio recupera su importancia y comienza a ser utilizado por la geografía, y en general por las ciencias sociales (Capel, 2016; Schneider y Peyré, 2006). A partir de ese momento se ha caracterizado por ser un concepto interdisciplinario, abierto, dúctil (Llanos-Hernández, 2010), general, transversal, multidimensional, complejo (Cabrera, 2016) y polisémico (Capel, 2016). En tal sentido, la multiplicidad de significados del término está asociada con diferentes perspectivas. Sólo por mencionar algunas, están la visión jurídica (Robledo-Silva, 2020), la económica (Cabrera, 2016), la social (Schneider y Peyré, 2006), la política (Sosa, 2012) y la cultural (Giménez, 2000). 
De las perspectivas antes mencionadas, interesa destacar la social. Según Schneider y Peyré (2006), el territorio tiene una proyección material y es al mismo tiempo "una construcción social del espacio que ocurre de forma colectiva entre los individuos e instituciones que están en el territorio y establecen códigos [para] pertenecer a éste" (p. 90). A dicha perspectiva es posible sumar la visión de la escuela francesa de la proximidad (école de la proximité), la cual entiende el territorio "más allá de la mera dimensión espacial de un espacio compartido entre actores y se convierte en un constructo social/institucional, ya que requiere que se involucren múltiples actores mediante diversas formas de coordinación" (Raufflet, 2017, p. 194).

Por otro lado, conviene señalar que el territorio incluye una amplia variedad de dimensiones que se asientan en diferentes perspectivas paradigmáticas y epistemológicas. Martínez (2012) afirma que el concepto no se limita a una dimensión geográfica y espacial, sino que también incluye las dimensiones económica, social, ambiental y organizativa. En la tabla 1 se esquematizan cuatro dimensiones del territorio y las formas de significarlo y analizarlo.

Tabla 1. Las dimensiones del territorio

\begin{tabular}{|c|c|c|}
\hline Dimensión & Significado & Se puede analizar como \\
\hline \multirow{3}{*}{ Social } & \multirow{3}{*}{$\begin{array}{c}\text { Relaciones y acciones } \\
\text { que establecen y realizan } \\
\text { los actores sociales }\end{array}$} & Proceso de organización \\
\hline & & Proceso de apropiación \\
\hline & & Construcción social \\
\hline \multirow{3}{*}{ Económica } & \multirow{3}{*}{$\begin{array}{l}\text { Características, dinámicas } \\
\text { y procesos económicos }\end{array}$} & Escenario de producción \\
\hline & & Lugar de producción \\
\hline & & Medio de producción \\
\hline \multirow{4}{*}{ Política } & \multirow{4}{*}{$\begin{array}{c}\text { Ejercicio de poder por la } \\
\text { posesión y el control del } \\
\text { territorio }\end{array}$} & Relaciones de dominio y poder \\
\hline & & Determinados intereses \\
\hline & & Institucionalidad (normas, instituciones) \\
\hline & & Políticas públicas (gobernanza) \\
\hline \multirow{3}{*}{ Cultural } & \multirow{3}{*}{$\begin{array}{l}\text { Un espacio con densidad } \\
\text { simbólica, actividad cultural } \\
\text { y contenido cosmogónico }\end{array}$} & Espacio de inscripción de la cultura \\
\hline & & Territorialidad cultural \\
\hline & & Elemento de identidad de un grupo \\
\hline
\end{tabular}

Fuente: López-Figueroa (2020, p. 13).

Al considerar el marco reflexivo anterior, conviene señalar que el territorio se puede comprender desde alguno de los diversos enfoques de los EO o se pueden aplicar conceptos teórico-metodológicos, como es el caso del campo organizacional (López-Figueroa, 2021). En la literatura sobre el concepto, se hallan muchas perspectivas teóricas que muestran variedad de significados para su entendimiento (Machado-da-Silva, Guarido y Rossoni, 2006). No obstante, en el ámbito de los EO se destacan tres perspectivas para entender el campo organizacional: la estructural, la cognitiva-cultural y la que está relacionada con el poder (Rosa, Pérez, Contreras y Hernández, 2017). 
La primera perspectiva - la estructural- se refiere a la noción clásica que proponen DiMaggio y Powell (1999), quienes definen el campo organizacional como "aquellas organizaciones que, en conjunto, constituyen un área reconocida de la vida institucional: los proveedores principales, los consumidores de recursos y productos, las agencias reguladoras y otras organizaciones que dan servicios o productos similares" (p. 106). Desde esta perspectiva, el proceso de estructuración o institucionalización de un campo consiste en las cuatro etapas que DiMaggio y Powell (1999) enuncian como sigue:

Un aumento en el grado de interacción entre las organizaciones en el campo; el surgimiento de estructuras interorganizacionales de dominio y de patrones de coalición claramente definidos; un incremento en la carga de información de la que deben ocuparse las organizaciones participantes de un campo; y el desarrollo de una conciencia entre los participantes de un conjunto de organizaciones que están involucrados en una empresa común. (p. 106)

La perspectiva cognitiva-cultural "denota la existencia de la comunidad de las organizaciones que forman parte de un sistema de significado común y cuyos participantes interactúan frecuente y fatídicamente más el uno con el otro que con los actores fuera del campo" (Scott, 2012b, p. 211). ${ }^{1}$ La tercera perspectiva visualiza el campo "como escenarios de relaciones de poder, en los que algunos actores, en general aquellos que poseen mayores recursos materiales $\mathrm{y} / \mathrm{o}$ simbólicos, tienen una posición de ventaja respecto a otros" (Brint y Karabel, 1999, p. 438). ${ }^{2}$

Para alejarse de las definiciones que tratan el campo organizacional como un conjunto construido por organizaciones, es necesario verlo como espacios de relaciones que tienen la virtud de brindarle a una organización la oportunidad de involucrarse con otros actores (Hoffman y Wooten, 2008). Este marco reflexivo es similar a entender el territorio desde la perspectiva de la escuela francesa de la proximidad, en la que se señala que en la dimensión espacial se involucran múltiples actores a través de diversas formas de relación y coordinación. Con estos argumentos es posible dar al territorio el trato de campo organizacional no para indicar que son en esencia la misma cosa, sino para subrayar que las organizaciones y sus procesos de relación y coordinación son un componente fundamental en la organización de los territorios.

Para ampliar la idea anterior, prestar atención a la diversidad de organizaciones que se integran en el territorio permite apreciar no sólo los fenómenos que están ligados al pensamiento económico, sino también otros procesos sociales, como las interacciones en las que el concepto de organización tiene un

1 En esta visión se puede ampliar el marco analítico del concepto para centrar la atención en la totalidad de los actores importantes, en las lógicas institucionales y en las estructuras de gobernanza que empoderan y restringen la acción de los participantes -tanto individuales como de las organizaciones- en un espacio social delimitado (Scott, 2014).

2 En las ciencias sociales, Bourdieu ha desarrollado el concepto de campo social no restringido a las organizaciones (citado en Scott, 2012b). Bajo esta concepción, se pueden visualizar las estrategias, los conflictos o los acuerdos entre actores, considerando la disponibilidad de capitales (económicos, culturales, simbólicos, sociales) en manos de ellos (Martínez, 2012). 
rol fundamental (López-Figueroa, 2021). De esta forma, López-Figueroa (2021) afirma lo siguiente:

El territorio, al ser definido como un campo organizacional, puede ser aprehendido desde la perspectiva de los estudios organizacionales a partir de sus enfoques o paradigmas resultando el concepto de organización fundamental para la comprensión y explicación de las relaciones sociales gestadas en los territorios. (p. 87)

DiMaggio (1999) ha estudiado los museos de arte como campo organizacional; Brint y Karabel (1999), las escuelas locales; Mazza y Pedersen (2004), el campo de la prensa; Reay y Hinings (2005), el cuidado de la salud y Coni-Zimmer (2014), la industria petrolera. En la región latinoamericana, Falcão-Vieira, Carvalho y Carvalho da Silva (2009) han estudiado los museos y teatros; Rivera (2018), la cinematografía; Sánchez (2020), el hospital psiquiátrico; Muñoz (2010), los residuos biológicos de los hospitales públicos; Ramos (2016), la educación superior municipal; Rosa, Pérez, Contreras y Hernández (2017), la cooperación internacional para el desarrollo y Silva (2016), la industria de textiles. Todas esas investigaciones ilustran la amplitud de sectores que pueden analizarse con el concepto de campo organizacional.

En el presente artículo se emplea la perspectiva de DiMaggio y Powell (1999) para describir la estructuración de un campo organizacional: la agricultura del valle del Yaqui en Sonora, como se mostrará en las siguientes líneas, no sin antes exponer una breve semblanza de ésta y sus múltiples estudios en relación con las dimensiones que configuran los territorios.

\section{El valle del Yaqui y sus estudios}

El territorio comprende tierras de los municipios de Guaymas, Empalme, Bácum, San Ignacio Río Muerto y Cajeme. Se localiza entre los paralelos $27^{\circ} 16^{\prime} 30^{\prime \prime}$ y $28^{\circ} 18^{\prime} 30^{\prime \prime}$ latitud norte y los meridianos $109^{\circ} 53^{\prime} 00^{\prime \prime}$ y $110^{\circ} 37^{\prime} 30^{\prime \prime}$ de longitud oeste de Greenwich, con una superficie estimada de 5500 kilómetros cuadrados $\left(\mathrm{km}^{2}\right)$ (López, 2019). Se compone de tres zonas con características geofísicas particulares. Según Olavarría (2000), éstas son: un área serrana, la sierra del Bacatete; una zona costera, que comprende poblados pesqueros, como Las Guásimas, Bahía de Lobos y Los Algodones; y el valle, donde se localizan las tierras irrigadas, los ocho pueblos cabecera (Bácum, Benaléi o Belén, Cócorit, Huirivis o Güíribis, Pótam, Rahúm, Tórim y Vícam) y más de cincuenta rancherías.

Mediante una revisión documental, se obtuvieron distintos datos que permiten señalar algunas de las características del valle.

1. La ocupación del suelo. Tiene como característica central la manzana y supone una extensión de 2000 metros por lado, lo que da una superficie de 400 hectáreas. Las manzanas, como se puede ver en la figura 2, consisten 
Figura 1. Territorio del valle del Yaqui

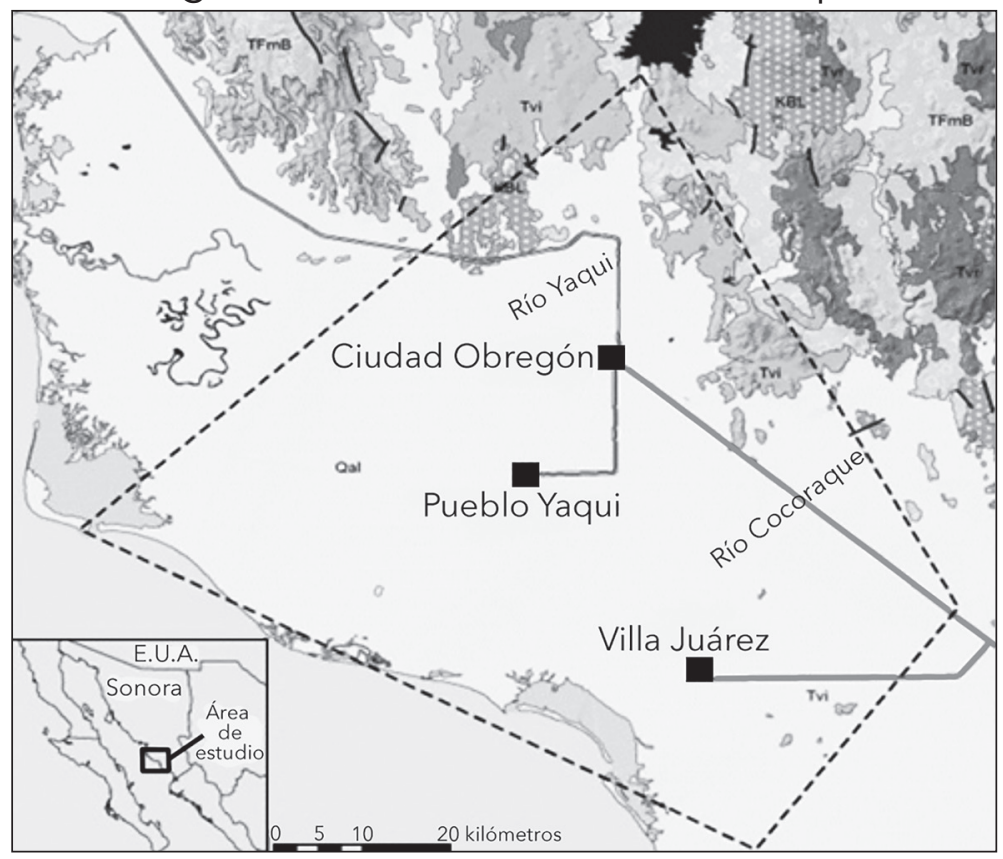

Fuente: Monreal, Rangel, Grijalva, Minjarez y Morales (2011, p. 125).

Figura 2. Trazado del valle del Yaqui

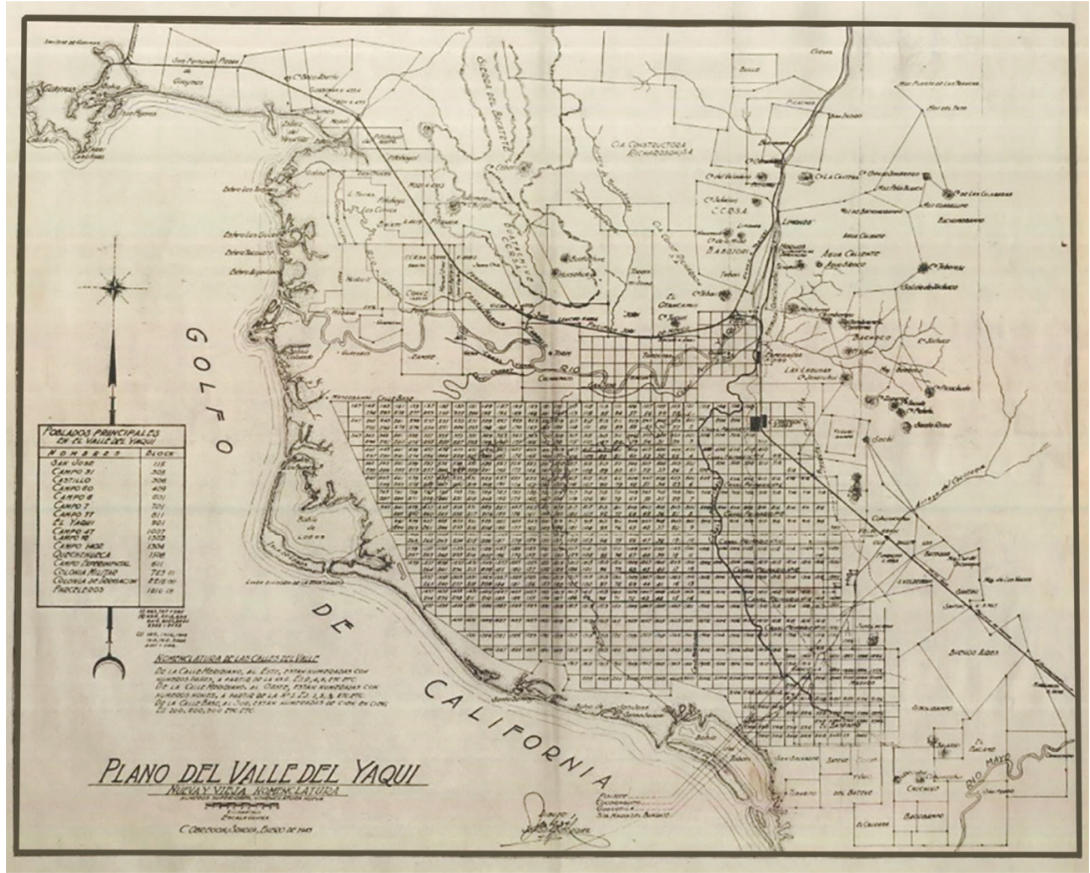

Fuente: Servicio de Información Agroalimentaria y Pesquera ([SIAP], 2016). 
en un sistema de cuadrículas divididas en 40 lotes de 200 por 500 metros, equivalentes a 10 hectáreas de superficie (Cerutti, 2019; Cerutti y Lorenzana, 2009).

2. La presa Oviáchic. Abastece $92 \%$ del agua al valle, y a las comunidades yaquis con 8\% (Méndez, Acosta, Mendoza, Soufflé, Oroz y Arreola, 2019).

3. Aprovechamientos subterráneos. Hay 39 de ellos. Son norias, pozos y manantiales. (Trasviña, Bórquez, Leal, Castro y Gutiérrez, 2018).

4. Riego por gravedad. El $96 \%$ del riego se hace por este medio; el resto corresponde a otros tipos de riego modernizado (Olmedo, 2017).

5. La revolución verde. El valle es el lugar de México donde comenzó dicha revolución (Cerutti, 2019; Cerutti y Lorenzana, 2009).

6. Cultivo. El principal es el trigo, que a escala nacional contribuye con $52 \%$ de la producción (Garatuza, Castro y Robles, 2017). Otros cultivos presentes en el territorio son el maíz, el cártamo, el cacahuate, la soya, el sorgo, el frijol y la alfalfa (Minjares y Félix, 2019).

7. Tipos de suelo. Aluvión ligero y pesado, barrial profundo, compactado y pedregoso (Olmedo, 2017).

8. Actores. El Centro de Investigaciones Agrícolas del Noroeste (CIRNO) y el Instituto Tecnológico de Sonora (ITSON), que llevan a cabo investigaciones agropecuarias (Cerutti, 2019).

9. Formas organizacionales. Corresponden a sociedades de producción rural (SPR), uniones de ejidos, uniones de crédito y las de representación regional y gestión de demandas (Almanza, 2008).

10. Tecnología. Uso considerable de tractores y trilladoras para trabajar la tierra (Cerutti, 2006).

Para ampliar el contexto del valle del Yaqui en cuanto a las formas de estudiarlo, a continuación se mencionan algunas investigaciones sobre las dimensiones territoriales que sugiere Sosa (2012): la social, la económica, la política y la cultural.

La primera se relaciona con la configuración del territorio, con las prácticas de apropiación por género y con las trayectorias y tendencias de ocupación. Desde esta dimensión, lo han estudiado Félix, Zúñiga y Soltero (2016) y Jiménez (2017).

La dimensión económica se relaciona con las formas de tenencia de la tierra, con las articulaciones productivas, con las redes de producción entre actores y con la posición geoestratégica. Desde esta dimensión, lo han estudiado Eichler, Kline, Ortiz-Monasterio, López-Ridaura y Dale (2020) y Ojeda, Mayer y Solomon (2008).

La dimensión política tiene que ver con las relaciones de poder para la apropiación del territorio, con el control sobre los medios de producción y con políticas públicas (Almanza, 2008; Cárdenas, 2016; Kumar, Lorek, Olsson, Sackley, Schmalzer y Soto, 2017).

La dimensión cultural se asocia con aspectos culturales, cosmogónicos e identitarios (Flores, Mungarro y García, 2021; Miner, 2021; Pubill y Buzinde, 2021). Por último, desde los estudios organizacionales, Flores, Ochoa y Jacobo 
(2020) y Ochoa, Cervantes, Jacobo y Flores (2020) se concentran en la innovación y el conocimiento.

\section{Método}

Los campos organizacionales - como todo sistema social-son abiertos. Por lo tanto, uno de los principales problemas que se presentan al estudiarlos consiste en definir sus límites, que resultan arbitrarios porque deben ser construidos (Mazza y Pedersen, 2004; Scott, 2014). La construcción de ellos puede incluir, como sugiere Scott (2014), el enfoque en los actores, en las actividades, en las relaciones o en los marcos cognitivos-culturales. A partir de este reconocimiento y bajo una exhaustiva revisión documental que considera el valle del Yaqui como uno de los territorios agrícolas más importantes de México y que reconoce la participación de diferentes actores locales, empresas, agencias gubernamentales, así como sociedades mercantiles y civiles, se determinó como criterio de selección la existencia de una práctica reconocida institucionalmente y la presencia de múltiples actores, los cuales se involucran mediante diversas formas de coordinación y de relación. Con base en estos criterios, se recabaron los datos de diferentes fuentes.

Se diseñaron dos entrevistas. La primera fue para los productores y aborda cuatro aspectos: los activos físico-territoriales (infraestructura productiva, recursos naturales y localización geográfica); los conflictos en relación con las instituciones gubernamentales (agua, medioambiente, suelo, clima y ubicación geográfica); la identificación de los actores (empresas, organizaciones, agencias gubernamentales y productores); y las relaciones entre los actores (comercialización de su cosecha, intercambio de productos, la compra de insumos para producir, la cooperación entre ellos y qué los hace estar en una red). La segunda fue para un asesor jurídico que abordó dos aspectos: la dotación de tierras en 1976 y la reforma al artículo 27 constitucional en 1992, con dos preguntas centrales: ¿cómo era el valle del Yaqui antes de la dotación y la reforma? y ¿qué cambió en el valle del Yaqui con la dotación y con la reforma? Esta entrevista resultó útil para identificar los actores existentes antes de la dotación de tierras en 1976, los que aparecieron con ella, los que desaparecieron con la reforma y los que aparecieron con ésta. Se trató de una entrevista complementaria para identificar o confirmar los actores que mencionaron los productores.

El resultado del trabajo de campo fueron 13 entrevistas a organizaciones ejidales, a arrendatarios (es decir, aquellos que pagan por usufructuar la tierra) y a un asesor jurídico. La duración promedio fue de 90 minutos. Se complementaron con tres notas de campo que permitieron reconocer y establecer las relaciones que los productores mantenían con las empresas que venden los insumos para producir y con aquellas que comercializan su producción. La investigación tuvo lugar en diferentes zonas del valle del Yaqui: en las parcelas de los productores entrevistados, en el campo 29 o Mora Villalobos, en el Campo 30 o Francisco I. Madero, en las oficinas de una unión de crédito, así como en los pueblos yaquis de Vícam y Pótam. El trabajo se realizó en dos etapas. La 
primera fase se hizo durante la preparación de la tierra. La segunda se llevó a cabo durante la época de la cosecha. Se estableció contacto con un productor arrendatario y él nos puso en contacto con otros, lo que en la investigación cualitativa se conoce como la técnica bola de nieve o en cadena. En la tabla 2 se muestran las características del trabajo de investigación.

Tabla 2. Características de la investigación

\begin{tabular}{|l|c|c|c|}
\hline \multicolumn{1}{|c|}{ Organización } & Extensión territorial & Entrevistas & Notas de campo \\
\hline Ejidal & 14 hectáreas & 1 & \\
\hline Ejidal & 100 hectáreas & 1 & 1 \\
\hline Arrendatario & 50 hectáreas & 2 & 2 \\
\hline Arrendatario & 230 hectáreas & 4 & \\
\hline Comunero de Vícam & 10 hectáreas & 1 & \\
\hline Arrendatario en Pótam y Vícam & 700 hectáreas & 1 & \\
\hline Arrendatario en Pótam & 1100 hectáreas & 2 & \\
\hline Asesor jurídico & - & 1 & \\
\hline Total & & 13 & 3 \\
\hline
\end{tabular}

Fuente: elaboración propia.

Con base en la información recopilada, se analizaron las cuatro etapas correspondientes a la estructuración de los campos organizacionales. Para dar respuesta a estas etapas, se construyeron ocho mapas agrupados en dos periodos: de 1970 a 1992 (antes de la reforma al artículo 27 constitucional) y a partir de 1992 (después de la reforma) hasta diciembre de 2020, cuando finalizó el estudio. Éstos se crearon para ambos periodos en los rubros: 1) de los activos físico-territoriales, 2) el de los conflictos, 3) el de los actores y 4) el de las redes o relaciones entre los actores. La intención fue analizar la información recogida durante el trabajo de campo, para reconstruir el valle del Yaqui de manera visual y saber si hubo cambios en las cuestiones físicas del territorio, en los conflictos, en los actores y en sus relaciones en los periodos mencionados. De manera particular, el mapa de los actores y el de las redes permitió encontrar atributos asociados con las cuatro etapas, con lo cual se pudo estructurar el valle del Yaqui como un campo organizacional construido por actores a partir de las relaciones que éstos establecen en el territorio.

\section{Resultados: la estructuración del campo organizacional del valle del Yaqui}

Una vez expuestas las consideraciones metodológicas, en esta sección se describe la estructuración del campo organizacional de la agricultura en el valle del Yaqui con base en las cuatro etapas que proponen DiMaggio y Powell (1999). 
Para ello, en primer lugar, se identifican a los actores integrantes de este territorio y, en segundo lugar, se presentan las cuatro etapas, ahondando en los actores y en las relaciones que ellos mantienen entre sí para conformar el campo agrícola.

Los actores del campo organizacional del valle del Yaqui

Por estar constituidos por los criterios relacionales y cognitivos de los actores, los campos organizacionales conducen a una cuestión: ¿son estables, o cambian a lo largo del tiempo? Ésta es una pregunta que Mazza y Pedersen (2004) se hacen cuando estudian la transformación de un campo organizacional. Ellos recopilan diversos factores de cambio, entre los que sobresalen los que sugieren DiMaggio y Powell (1999). El cambio en los campos está determinado por su ciclo de vida, de forma tal que en los campos jóvenes el cambio proviene de las fuerzas económicas y competitivas, mientras que en los campos maduros ${ }^{3}$ hay una estabilidad derivada del isomorfismo institucional (DiMaggio y Powell, 1999; Mazza y Pedersen, 2004).

Para estructurar el campo organizacional, es necesario, en primer lugar, identificar los actores. Bajo la idea de cambio, en el valle del Yaqui es posible ubicar la participación de varios de ellos. Los cambios se pueden extender a tres periodos: el primero consiste en la creación de los ejidos y se ubica entre los años 1930 y 1970. En la década de 1930, la concentración de grandes extensiones de tierra estaba en pocas manos. En un relato de Uribe-García (1994) se dice que 85 miembros de la élite colonizadora del valle eran propietarios de más de 28000 hectáreas de riego, mientras que las otras 16000 hectáreas de riego estaban distribuidas en propiedades de 150 hectáreas o menos.

La concentración de tierras en propiedades que sobrepasaban el límite de 100 hectáreas, causó la expropiación oficial del valle del Yaqui en 1937 durante la administración de Lázaro Cárdenas, la cual "trató de reducir el poder económico y político de la élite terrateniente del valle del Yaqui [...] mediante la entrega de estas tierras a los campesinos en forma de ejidos colectivos" ( $p$. 215). En el marco de la expropiación, los primeros núcleos de población dotados de manera provisional fueron: Cócorit, Esperanza, Providencia, Campo 700, Campo 77, El Castillo, Campo Yaqui, Campo 47, Campo 16, Cajeme, Campo 60, Quetchehueca y Campo 1402 (Lorenzana, 2017).

En la década de 1950 irrumpió, bajo la influencia de diferentes actores, sobre todo de Norman E. Borlaug, la denominada revolución verde que nació, según Cerutti (2019), debido a la aplicación de un paquete tecnológico que incluía una investigación aplicada sobre las variedades de trigo, las semillas mejoradas, la combinación de la irrigación, la mecanización, los fertilizantes y

3 Una diferencia entre los campos organizacionales jóvenes y los maduros es que los últimos tienen homogeneidad estructural en las organizaciones integrantes del campo. Tal homogeneidad se recoge en el concepto de isomorfismo institucional propuesto por DiMaggio y Powell y se refiere al parecido estructural que presentan las organizaciones del campo. En cambio, en los campos jóvenes, la homogeneidad o isomorfismo institucional no está del todo consolidada, por lo que en esos campos las fuerzas económicas (agentes, sistemas, actores económicos) desempeñan un papel importante en la estructuración del campo. 
los insecticidas, entre otros insumos. Con dicha revolución aparecieron actores dedicados a la investigación agropecuaria y a la formación de recursos humanos - como es el caso del CIRNO, del ITSON y del Centro Regional Universitario del Noroeste (CRUNO) - los cuales establecen un vínculo entre el Estado, la investigación científica, los sectores productivos y la universidad (Ceruti, 2019).

El segundo periodo consiste en la consolidación de los ejidos colectivos y se puede ubicar entre 1971 y 1992. El artículo 27 constitucional, antes de la reforma de 1992, señalaba que los campesinos, los núcleos de población o los grupos de personas tenían derecho de petición de tierra por la vía de nuevos centros de población, dotación o ampliación. En el caso del valle del Yaqui, bajo este derecho de petición, se fue solicitando tierra de los pequeños propietarios. Así, con el esquema de nulidad de fraccionamientos simulados, en octubre de 1975, en San Ignacio Río Muerto, se afectaron 5700 hectáreas. Un año después, el 19 de noviembre de 1976, se afectaron, según la información de las entrevistas, 37600 hectáreas con el mismo esquema de 1975. Un relato similar se encuentra en Otero (2004) cuando dice que "el 19 de noviembre de 1976, es decir, pocos días antes de concluir el periodo presidencial de Luis Echeverría, fueron expropiadas casi 100000 hectáreas de los valles del Yaqui y el Mayo. Afectando un total de 40 por ciento de las tierras de propiedad privada en el valle del Yaqui" (p. 199).

Hasta donde mostró el trabajo de campo, se entregaron mediante la expropiación 37000 hectáreas de tierras irrigables que conformaron 76 ejidos colectivos. Como consecuencia, la tierra pasó de estar en pocas manos a distribuirse entre un gran número de campesinos. En este contexto, y con apoyo de los mapas, es posible mencionar, además del ejido y de los actores dedicados a la investigación agropecuaria y a la formación de recursos humanos, a otros actores que se ubican en los periodos mencionados: pequeños propietarios, colonos, comunidades yaquis, uniones de crédito, uniones de ejidos, asociaciones rurales de interés colectivo, organizaciones privadas, la Compañía Nacional de Subsistencias Populares (CONASUPO), la Aseguradora Nacional Agrícola y Ganadera (ANAGSA), la Secretaría de Agricultura y Recursos Hidráulicos (SARH), el Banco de Desarrollo Rural (BANRURAL) y Fideicomisos Instituidos en Relación con la Agricultura (FIRA).

El tercer periodo consiste en la decadencia del ejido y la mercantilización de la tierra, ubicado después de la reforma al artículo 27 constitucional en 1992. En aquel tiempo, Gómez de Silva (2016) refiere que se estaba buscando una transformación del campo mexicano debido a la falta de certeza en la tenencia de la tierra, a la existencia del minifundio -en las distintas formas de propiedad-, al estancamiento de los rendimientos y a la baja rentabilidad de muchos cultivos. Estas problemáticas - continúa el autor- hicieron indispensable que a la inversión del sector público se sumara la del sector privado.

La reforma al artículo 27 constitucional consideró poner fin al reparto agrario bajo la justificación de un agotamiento de tierras, de la urgencia de capitalizar el campo para hacerlo productivo y competitivo, de lograr la plenitud jurídica, así como de regularizar y certificar la propiedad agraria para alcanzar la identidad jurídica y geográfica (Rivera-Rodríguez, 2016). De manera complementaria, en la década de 1990 se establecieron otras instituciones que 
servirían de sostén para la mercantilización de la tierra. En el decreto del 23 de febrero de 1992, publicado el 26 de ese mes y año, se expidió la Ley Agraria como reglamentaria del artículo 27 constitucional (Gómez de Silva, 2016). De esta forma concluye el reparto agrario y se autoriza a la asamblea ejidal otorgar el dominio pleno sobre sus parcelas a los ejidatarios y culmina la forma en que el Estado venía apoyando a los campesinos en el proceso de producción (González-Navarro, 2016).

La reforma de 1992 y la nueva Ley Agraria respaldaron la llegada de nuevos actores. Con ayuda de los mapas, es posible mencionar otra diversidad de actores que se ubican en este periodo: las sociedades mercantiles y civiles, las sociedades de producción rural, los productores independientes, los arrendatarios, las empresas medianas o grandes, las uniones de crédito, las parafinancieras, los fondos de aseguramiento, las empresas que compran la producción, la financiera rural y los bancos privados.

La construcción de mapas por periodos permitió identificar a los actores que se integran en el territorio. Sin embargo, vale decir que para el tercer periodo algunos de ellos ya habían desaparecido, como BANRURAL, CONASUPO y Fertilizantes de México (FERTIMEX). Otros evolucionaron, como la SARH, que se transformó en la Secretaría de Agricultura, Ganadería, Desarrollo Rural, Pesca y Alimentación (SAGARPA), hoy Secretaría de Agricultura y Desarrollo Rural (SADER). La Aseguradora Nacional de la Agricultura y Ganadería (ANAGSA) desapareció y dio lugar al surgimiento de Agroasemex, Sociedad Anónima (S. A.). Los actores que se mantienen en estos periodos son FIRA, Financiera Nacional de Desarrollo (FND), los ejidos, los pequeños propietarios y los colonos. Como nuevos actores identificados, están los fondos de aseguramiento, las uniones de crédito, las parafinancieras, las sociedades de producción rural, los arrendatarios, las sociedades civiles, las sociedades mercantiles y la banca privada.

Tomando como base la argumentación anterior, y con la intención de dar mayor rigor y sentar las bases de la participación de los actores en el territorio, a continuación se sugiere una tipología de los actores organizacionales en el valle del Yaqui. En el primer nivel están las organizaciones productoras, que son aquellas que se relacionan de forma directa con la producción agrícola. Entre ellas se encuentran las personas físicas, los ejidos, los colonos, los pequeños propietarios, las sociedades de producción rural y los arrendatarios. En el segundo nivel están las organizaciones de grupos de productores, que son las asociaciones de productores (ejidos, personas físicas, pequeños propietarios, colonos y arrendatarios) que forman sociedades, parafinancieras, uniones de crédito, uniones de ejidos o fondos de aseguramiento. Este tipo de asociaciones tiene como finalidad brindar servicio de financiamiento, asistencia técnica, insumos y canales de comercialización. ${ }^{4}$ En el tercero están las organizaciones de

4 En el valle del Yaqui es posible identificar dos formas de organización: por un lado, aquellos productores que trabajan de manera independiente y, por el otro, aquellos que trabajan de manera colectiva o en asociación. Los primeros buscan por sus propios medios los recursos para producir, qué producir, cómo producir, la asistencia técnica, etcétera. En cambio, los colectivos son aquellos que deciden conformar o agremiarse a las uniones de crédito o las parafinancieras para obtener mejores condiciones en cuanto a asistencia técnica, comercialización, insumos y financiamiento. Es por lo anterior que el lector podrá encontrar actores individuales en las primeras dos tipologías de organización. 
no productores pero vinculadas a los productores. Son aquellas organizaciones privadas que prestan distintos servicios: económicos, insumos, laboratorios de investigación y empresas que compran la producción. En el cuarto están las organizaciones políticas, que dependen de manera directa de las autoridades gubernamentales, como la SADER. En el quinto están las organizaciones de financiamiento de carácter gubernamental, que están dedicadas al financiamiento del campo, pero como banca de segundo piso. Entre ellas se hallan FIRA y FND. En el último nivel se sitúan las organizaciones de financiamiento de carácter privado, que componen la banca privada y prestan servicios financieros al campo. Esta identificación de actores permite estructurar el campo organizacional. Sin embargo, se ha decidido aquí ampliar la visión sobre la estructuración del campo a partir de las relaciones que establecen los actores en el territorio, como se verá en la siguiente sección.

La estructuración del campo organizacional del valle del Yaqui

La estructura de un campo no puede determinarse a priori, sino que debe definirse con base en la investigación empírica (DiMaggio y Powell, 1999). Conforme a los planteamientos de la sección anterior, en la figura 3 se presenta la estructura del campo organizacional.

Figura 3. Estructura del campo organizacional de la agricultura del valle del Yaqui

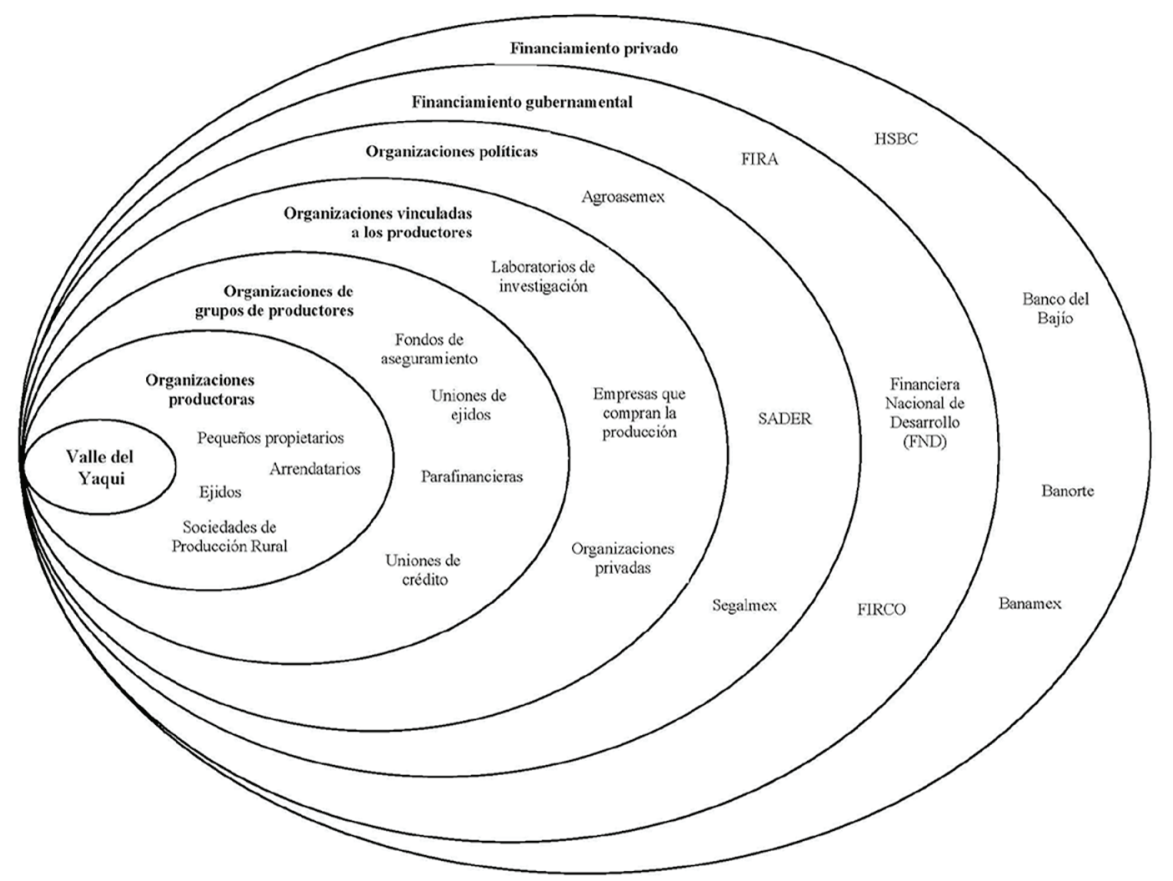

Fuente: elaboración propia. 
Desde la posición de DiMaggio y Powell (1999), el proceso para alcanzar la estructuración del campo de la agricultura en el valle del Yaqui implica dar cuenta "del aumento en el grado de interacción entre las organizaciones en el campo" (p. 106). Una de las formas en que las organizaciones productoras interaccionan es a través de la asociación para conformar las organizaciones de grupos de productores, como ya se señaló. En las sociedades, se da la rendición de cuentas para revisar los estados financieros y determinar qué cultivos sembrar y dónde comercializar. También, entre las organizaciones productoras, se realizan convenios para facilitar los procesos de comercialización y crédito. Así mismo la interacción aumenta cuando hay pactos con organizaciones políticas, por lo general, con el gobierno federal debido a los programas de apoyo que éste otorga para obtener maquinaria, sistemas de riego y subsidios o ayuda para determinados cultivos.

Estructurar el campo implica dar cuenta del "surgimiento de estructuras interorganizacionales de dominio y de patrones de coalición claramente definidos" (p. 106). Para satisfacer esta etapa, es necesario considerar las organizaciones de grupos de productores. Este tipo de actor responde a intereses colectivos a través de un enfoque integral de financiamiento, asistencia técnica, insumos y canales de comercialización, los cuales conforman una red con intereses diferentes que se extiende a todas las actividades durante el ciclo agrícola. Las estructuras de dominación también se visualizan cuando los productores independientes deciden agremiarse en este tipo de organizaciones donde negocian intereses individuales para fortalecer sus capacidades. De esta manera, estos actores logran obtener mejores condiciones. En este sentido, las organizaciones de grupos de productores también permiten aumentar la intensidad de interacción entre actores para conformar redes.

El reconocimiento de los actores, su grado de interacción y las estructuras de dominación permiten sostener que el campo organizacional se está estructurando, aunque aún no esté bien definido. Todavía falta dar cuenta de "un incremento en la carga de información de la que deben ocuparse las organizaciones que participan en un campo" (DiMaggio y Powell, 1990, p. 106). En cuanto a esta etapa, es importante mencionar la generación de convenios con el gobierno federal, con empresas privadas para comercializar la producción, entre productores, con la Comisión Nacional del Agua o los distritos de riego, así como las relaciones con la Financiera Nacional de Desarrollo o las uniones de crédito, la asistencia técnica pública o privada y la investigación agropecuaria que realizan el CIRNO y el ITSON. Todos estos aspectos aumentan los flujos de información en el campo organizacional.

Sólo falta dar cuenta del "desarrollo de la conciencia entre los participantes de un conjunto de organizaciones que están en una empresa común" (DiMaggio y Powell, 1990, p. 106). Como se ha señalado antes, los cambios que ha tenido el valle del Yaqui ubican la participación de diversos actores. Aun cuando todos ellos estén interactuando y creando procesos de confianza o una mutua conciencia, no significa que tengan una empresa en común (Myers, 2014). Para entender la conciencia mutua en el campo organizacional, el pilar cognitivo de las instituciones resulta de utilidad por "resaltar el rol central que ocupa la 
construcción socialmente mediada de un esquema de significado común" (Scott, 2012b, p. 199).

La base legítima de este pilar proviene de adoptar un marco común de referencia. En tal sentido, para aumentar la conciencia mutua, los actores del campo requieren un lenguaje o un sistema de significado cuyos actores deben compartir entre sí. Los actores antiguos, como los ejidos y los pequeños propietarios, o los actores con gran trascendencia en el territorio, como las parafinancieras y las uniones de crédito que agremian a diferentes actores, tienen los conocimientos y las capacidades para crear el significado o un lenguaje en común, pues ellas transfieren información, recursos e insumos a sus agremiados. De esta forma, las asociaciones refuerzan la conciencia de los actores productivos haciéndolos parte de una empresa en común. Con todo lo anterior, los actores del campo organizacional realizan esfuerzos de relación conjunta o de asociación para lograr un territorio organizado.

Estructurar el campo organizacional de la agricultura en el valle del Yaqui ha implicado revisar su conformación socio-histórica, es decir, sus cambios a través del tiempo. Bajo la idea de campo organizacional, en este territorio aparecen actores clave, como las organizaciones de grupos de productores, quienes desarrollan actividades para coordinar las acciones de otros actores, con lo cual logran la estructuración del campo como consecuencia de las interacciones, las relaciones y las actividades. Así, se ha podido reconocer un componente de la vida institucional: la agricultura del sur de Sonora.

\section{Conclusiones}

Este trabajo es un esfuerzo para estructurar el campo organizacional de la agricultura en el valle del Yaqui, utilizando los argumentos no sólo de DiMaggio y Powell (1999), quienes visualizan el campo como la totalidad de los actores importantes, sino también subrayando los significados, las acciones de dichos actores, las relaciones y las estructuras de gobernanza, como sugiere Scott (2014), para conformar los campos organizacionales. La esencia del campo organizacional -en cuanto que constructo analítico- consiste en su capacidad para servir como un lugar de encuentro donde las organizaciones tienen la oportunidad de realizar procesos de negociación, comunicación, asociación y organización, entre otros (Hoffman y Wooten, 2008).

Abordar el estudio del territorio desde la perspectiva de los EO, implica considerarlo como un campo organizacional y éste como un espacio relacional para destacar el papel de las organizaciones en él. No obstante, Aubert, Guérin y Perrier-Cornet (2001) argumentan que la noción de organización no es propia de la categoría de territorio o espacio. Sin embargo, sus alcances permiten estudiar ciertos fenómenos, como las formas de coordinación o de relación, y no sólo centrarse en el fenómeno del desarrollo.

Considerando la argumentación anterior, visualizar el territorio desde la perspectiva de los EO no sólo permite el análisis a nivel de las organizaciones sino, también, indagar en otras cosas de carácter local, como las instituciones 
(Hodgson, 2011), la identidad organizacional (Albert y Whetten, 1985), la gobernanza y los costos de transacción (Helmsing y Ellinger, 2011; Williamson, 2002), las reglas (March y Olsen, 1997), los sistemas de significados (Meyer y Rowan, 1999), el campo organizacional y el isomorfismo -coercitivo, mimético y normativo (DiMaggio y Powell, 1999)-, las lógicas institucionales (Tolbert y Zucker, 1996) y las relaciones de poder (Crozier y Friedberg, 1990). Todo esto permite ubicar no sólo a los actores referidos (instituciones, organizaciones, individuos, sujetos), sino también comprender la complejidad territorial compuesta de distintos elementos organizacionales (Contreras, 2017).

Hay que concentrarse, pues, en comprender el campo organizacional como una unidad de análisis para intervenir los territorios a partir de un componente clave de los campos: los sistemas relacionales (Scott, 2014). Ese componente consiste en la asociación de organizaciones en redes. Los sistemas se recogen en la gobernanza, concepto que Prats (2003) define como los procesos de interacción entre actores estratégicos, de los cuales emergen estructuras sociopolíticas que forjan procesos de interacción reflexiva. Siguiendo este razonamiento, la gobernanza resulta ser una herramienta analítica y descriptiva, porque los patrones de interacción son lo que son, aunque también son el resultado de la acción de los actores y tienen una dimensión normativa.

De acuerdo con Scott (2014), cada campo organizacional se caracteriza por tener un sistema de gobernanza en particular compuesto de actores públicos y privados, quienes emplean una combinación de controles normativos y regulatorios sobre las actividades y los mismos actores dentro del campo. En este caso de estudio, los actores que ejercen estas funciones son las organizaciones de productores, las organizaciones de grupos de productores, las organizaciones de no productores pero vinculadas a los productores, las organizaciones políticas, las organizaciones de financiamiento de carácter gubernamental y las organizaciones de financiamiento de carácter privado, como se aprecia en la figura 3.

Aquí se propone la gobernanza como una estrategia para la intervención de los territorios que permita describir los procesos de interacción entre los diferentes actores, las formas y las modalidades para tomar decisiones y saber cómo se ejerce el poder. Brenner y Rosales (2015) sostienen que es posible adoptar por lo menos tres diferentes maneras de percibirla y analizarla en el territorio: la primera consiste en el enfoque descriptivo-analítico, que centra su análisis en los actores, sus interacciones y las relaciones de poder para comprender aspectos territoriales, como la organización económica, las cadenas de valor, el desarrollo económico local y las certificaciones como requisitos para participar en el mercado. La segunda consiste en la conceptualización normativa (o idealista) de la gobernanza, que incorpora los principios de buena gobernanza que aconsejan algunos organismos internacionales, como el Banco Mundial y el Fondo Monetario Internacional, para comprender ciertos aspectos, como los servicios ambientales, la certificación de productos y la promoción de la sustentabilidad ambiental y la justicia social. La tercera consiste en la visión crítica de la buena gobernanza para imponer regímenes de gobernanza e implementar políticas neoliberales. Mediante esta estrategia de intervención, es 
posible comprender algunos aspectos locales de los territorios que contribuyan al diseño de políticas públicas para el desarrollo local.

En suma, los avatares que enfrentan los territorios mexicanos requieren paradigmas alternativos asentados en una postura analítica y comprensiva. Es aquí donde los estudios organizacionales y sus líneas de investigación -entre ellas, el institucionalismo, la gobernanza, la intervención organizacional, el género y el poder - tienen la oportunidad de convertirse en una alternativa que amplíe el ángulo de análisis para la comprensión de los territorios.

\section{Referencias}

Albert, S., y Whetten, D. (1985). Organizational identity. Research in Organizational Behavior (7), 263-295.

Almanza, M. (2008). Las organizaciones del sector social del valle del Yaqui. Retrocesos de política agraria. Frontera Norte, 20(40), 135-167. doi: https:// doi.org/https://doi.org/10.17428/rfn.v20i40.988

Aubert, F., Guérin, M., y Perrier-Cornet, P. (2001). Organisation et territoire: un cadre d'analyse appliqué aux espaces ruraux. Evue d'Économie Régionale y Urbaine (3), 393-413. doi: https://doi.org/10.3917/reru.013.0393

Brenner, L., y Rosales, R. (2015). Introducción. Procesos de construcción de gobernanza. En R. Rosales y L. Brenner (eds.), Geografía de la gobernanza: dinámicas multiescalares de los procesos económico-ambientales (pp. 7-19). Ciudad de México: Universidad Autónoma Metropolitana (UAM)-Iztapalapa y Siglo XXI Editores.

Brint, S., y Karabel, J. (1999). Los orígenes y las transformaciones institucionales: el caso de las escuelas locales de los Estados Unidos. En W. Powell y P. DiMaggio (eds.), El nuevo institucionalismo en el análisis organizacional (pp. 413-440). Ciudad de México: Colegio Nacional de Ciencias Políticas y Administración Pública, Universidad Autónoma del Estado de México y Fondo de Cultura Económica (FCE).

Cabrera, V. (2016). El nodo territorio-epistemología. Ausencias, presencias e implicaciones. En V. Cabrera y E. Licona (eds.), Para pensar el territorio. Elementos epistemológicos y teóricos (pp. 7-20). Puebla: Benemérita Universidad Autónoma de Puebla (BUAP).

Capel, H. (2016). Las ciencias sociales y el estudio del territorio. Biblio3W, 21(1.149), 1-38. Recuperado de http://www.ub.edu/geocrit/b3w-1149.pdf

Cárdenas, N. (2016). Movimiento campesino y oportunidades de cambio político y social. La experiencia del valle del Yaqui (1920-1950). Estudios de Historia Moderna y Contemporánea de México (52), 69-87. doi: https:// doi. org/10.1016/j.ehmcm.2016.06.002

Cerutti, M. (2006). La construcción de una agrociudad en el noroeste de México. Ciudad Obregón (1925-1960). En A. Carrillo y M. Cerutti (eds.), Agricultura comercial, empresa y desarrollo regional en el noroeste de México (pp. 87-120). Culiacán: Universidad Autónoma de Sinaloa. 
Cerutti, M. (2019). Trigo y revolución verde en el noroeste de México (1930-1970). Mundo Agrario, 20(43), 1-21. doi: https: // doi.org/10.24215/15155994e103

Cerutti, M., y Lorenzana, G. (2009). Irrigación, expansión de la frontera agrícola y empresariado en el Yaqui (1925-1965). América Latina en la Historia Económica (31), 5-36. doi: https://doi.org/10.18232/alhe.v16i1.403

Coni-Zimmer, M. (2014). Organizational fields, transnational business governance interactions and the diffusion of CSR. Osgoode Legal Studies Research Paper Series, 10(7). Recuperado de https: / / digitalcommons.osgoode.yorku. $\mathrm{ca} /$ olsrps/48/

Contreras, R. (coord.) (2017). Territorio y organizaciones. En D. Herrera, G. Ramírez y J. Rosas (coords. grales.), Diversidad y complejidad organizacional en América Latina. Perspectivas de análisis. Ciudad de México: Grupo Editorial Hess. Recuperado de http://remineo.org/repositorio/libros/dcoal/ libros/territorio-y-organizaciones/

Crozier, M., y Friedberg, E. (1990). El actor y el sistema. México: Alianza Editorial.

DiMaggio, P. (1999). Construcción de un campo organizacional como un proyecto profesional: los museos de arte de los Estados Unidos, 1920-1940. En W. Powell y P. DiMaggio (eds.), El nuevo institucionalismo en el análisis organizacional (pp. 333-361). México: Colegio Nacional de Ciencias Políticas y Administración Pública, Universidad Autónoma del Estado de México y FCE.

DiMaggio, P., y Powell, W. (1999). Retorno a la jaula de hierro: el isomorfismo institucional y la racionalidad colectiva en los campos organizacionales. En W. Powell y P. DiMaggio (eds.), El nuevo institucionalismo en el análisis organizacional (pp. 104-125). Ciudad de México: Colegio Nacional de Ciencias Políticas y Administración Pública, Universidad Autónoma del Estado de México y FCE.

Eichler, S. E., Kline, K. L., Ortiz-Monasterio, I., López-Ridaura, S., y Dale, V. H. (2020). Rapid appraisal using landscape sustainability indicators for Yaqui Valley, Mexico. Environmental and Sustainability Indicators, 6(100029). doi: https://doi.org/10.1016/j.indic.2020.100029

Falcão-Vieira, M., Carvalho, C., y Carvalho da Silva, R. (2009). The process of historic evolution of organizational fields: an analysis of museums and theatres in southern and northeastern Brazil. International Journal of Arts Management, 11(2), 20-28. Recuperado de http:/ / www.jstor.org/stable/41064984

Félix, H., Zúñiga, H., y Soltero, M. (2016). Aproximaciones a la cotidianidad de las mujeres del valle del Yaqui, Sonora, México. Culturales, 4(2), 103-133. Recuperado de http://www.scielo.org.mx/scielo.php?script=sci_arttext\&pi$\mathrm{d}=\mathrm{S} 1870-11912016000200103 \& \operatorname{lng}=e s \& t \operatorname{lng}=e s$

Flores, J., Mungarro, Z., y García, R. (2021). Elements of Yaqui culture as an ethnoturistic attractive in Sonora, Mexico. Orbis (48), 20-34. Recuperado de http: / / www. revistaorbis.org/pdf/53/art2.pdf

Flores, J., Ochoa, S., y Jacobo, C. (2020). Knowledge management and innovation in agricultural organizations: an empirical study in the rural sector of northwest Mexico. Cuadernos de Desarrollo Rural, 17(86). doi: https://doi. org/10.11144/Javeriana.cdr17.kmia 
Garatuza, J., Castro, J., y Robles, A. (2017). Expansión urbana de Ciudad Obregón en relación con la tenencia de la tierra en el valle del Yaqui, Sonora. Avances de Investigación. I Encuentro Científico y Técnico.

Giménez, G. (2000). Territorio, cultura e identidades. La región socio-cultural. En R. Rosales Ortega (ed.), Globalización y regiones en México (pp. 19-51). Ciudad de México: Universidad Nacional Autónoma de México (UNAM).

Gómez de Silva, J. (2016). El derecho agrario mexicano y la Constitución de 1917. México: Secretaría de Gobernación, Secretaría de Cultura, Instituto Nacional de Estudios Históricos de las Revoluciones de México, UNAM-Instituto de Investigaciones Jurídicas.

González-Navarro, G. (2016). El derecho agrario a la luz del artículo 27 constitucional. En E. Ferrer y L. R. Guerrero (coords.), Derechos del pueblo mexicano. México a través de sus constituciones (pp. 566-577). México: Cámara de Diputados del H. Congreso de la Unión, LXIII Legislatura, Suprema Corte de Justicia de la Nación, Instituto Nacional Electoral, Comisión Nacional de los Derechos Humanos, Senado de la República, LXIII Legislatura, Tribunal Electoral del Poder Judicial de la Federación y Miguel Ángel Porrúa.

Helmsing, A. H. J., y Ellinger, P. (2011). La economía política institucional del desarrollo local: dos cuentos de turismo en Brasil. EURE-Revista de Estudios Urbano Regionales, 37(110), 37-51. doi: https://doi.org/10.4067/S025071612011000100002

Hodgson, G. (2011). ¿Qué son las instituciones? CS (8), 17-53. Recuperado de https://www.icesi.edu.co/revistas/index.php/revista_cs/article/view/1128

Hoffman, A., y Wooten, M. (2008). Organizational fields: past, present and future. En R. Greenwood, C. Oliver, K. Sahlin y R. Suddaby (eds.), The Sage Handbook of Organizational Institutionalism (pp. 56-74). Londres: Sage Publications.

Jiménez, E. (2017). Género, etnia y globalización: identificación y análisis de tres estrategias que realizan las mujeres yaquis dentro de sus hogares para ganar autonomía. Frontera Norte, 25(49), 131-156. doi: https://doi. org/10.17428/rfn.v25i49.795

Kumar, P., Lorek, T., Olsson, T., Sackley, N., Schmalzer, S., y Soto, G. (2017). Roundtable: new narratives of the green revolution. Agricultural History, 91(3), 397-422. doi: https://doi.org/10.3098/ah.2017.091.3.397

Llanos-Hernández, L. (2010). El concepto del territorio y la investigación en las ciencias sociales. Agricultura, Sociedad y Desarrollo, 7(3), 207-220. Recuperado de http://www.scielo.org.mx/pdf/asd/v7n3/v7n3a1.pdf

López-Figueroa, J. C. (2020). El concepto de territorio en el escenario de la perspectiva organizacional. Gestión y Estrategia (58), 9-24. Recuperado de http://gestionyestrategia.azc.uam.mx/index.php/rge/article/view/700

López-Figueroa, J. C. (2021). Pensar el territorio desde los estudios organizacionales: conversaciones para el análisis organizacional. Innovar, 31(79), 79-92. doi: https://doi.org/10.15446/innovar.v31n79.91891

López, R. (2019). Contexto y estrategia para el desarrollo económico y social de la tribu yaqui. En J. Arreola, J. Garatuza, E. Yépez y A. Robles (eds.), Ca- 
pital natural y bienestar social de la comunidad yaqui (pp. 366-383). Ciudad Obregón: Instituto Tecnológico de Sonora.

Lorenzana, G. (2017). El reparto agrario en el valle del Yaqui, Sonora: un diferendo diplomático entre México y los Estados Unidos, 1936-1938. HiSTOReLo, 9(17), 442-470. doi: https://dx.doi.org/10.15446/historelo.v9n17.55506

Machado-da-Silva, C. L., Guarido Filho, E. R., y Rossoni, L. (2006). Organizational fields and the structuration perspective: analytical possibilities. BAR-Brazilian Administration Review, 3(2), 32-56. doi: https://doi.org/10.1590/ s1807-76922006000200004

March, J., y Olsen, J. (1997). El redescubrimiento de las instituciones. La base organizativa de la política. México: FCE, Colegio Nacional de Ciencias Políticas y Administración Pública y Universidad Autónoma de Sinaloa.

Martínez, L. (2012). Apuntes para pensar el territorio desde una dimensión social. Ciências Sociais Unisinos, São Leopoldo, 48(1), 12-18. doi: https:// doi. org/10.4013/csu.2012.48.1.02

Mazza, C., y Pedersen, J. S. (2004). From press to e-media? The transformation of an organizational field. Organization Studies, 25(6), 875-896. doi: https: / / doi.org/10.1177/0170840604042407

Méndez, L., Acosta, B., Mendoza, R., Soufflé, L., Oroz, L., y Arreola, J. (2019). Abastecimiento y calidad del agua superficial y subterránea. En J. Arreola, J. Garatuza, E. Yépez y A. Robles (eds.), Capital natural y bienestar social de la comunidad yaqui (pp. 135-160). Ciudad Obregón: Instituto Tecnológico de Sonora.

Meyer, J., y Rowan, B. (1999). Organizaciones institucionalizadas: la estructura formal como mito y ceremonia. En W. Powell y P. DiMaggio (eds.), El nuevo institucionalismo en el análisis organizacional (pp. 79-103). México: Colegio Nacional de Ciencias Políticas y Administración Pública, Universidad Autónoma del Estado de México y FCE.

Miner, J. (2021). Ethnographic photobomb: the materiality of decolonial image manipulation. International Journal of Cultural Studies, 24(3), 414-433. doi: https: / / doi.org/10.1177/1367877920976654

Minjares, J., y Félix, J. (2019). Desarrollo de la agricultura en la tribu yaqui. En J. Arreola, J. Garatuza, E. Yépez y A. Robles (eds.), Capital natural y bienestar social de la comunidad yaqui (pp. 162-184). Ciudad Obregón: Instituto Tecnológico de Sonora.

Monreal, R., Rangel, M., Grijalva A., Minjarez, I., y Morales, M. (2011). Metodología para la definición de unidades hidroestratigráficas: caso del acuífero del valle del río Yaqui, Sonora, México. Boletín de la Sociedad Geológica Mexicana, 63(1), 119-135. Recuperado de https://www.redalyc.org/articulo. oa?id=94319372011

Montaño, L. (2020). Encrucijadas y desafíos de los estudios organizacionales. Una reflexión desde las perspectivas institucionales. Innovar, 30(78), 19-34. doi: https: / / doi.org/10.15446/innovar.v30n78.90304

Muñoz, R. (2010). Los campos organizacionales de los residuos biológicos en los hospitales públicos. Iztapalapa, Revista de Ciencias Sociales y Humanidades (68), 155-180. Recuperado de http://www.redalyc.org/articulo. oa?id $=39348725008$ 
Myers, V. (2014). Conversations about Calling: Advancing Management Perspectives. Nueva York: Routledge. doi: https://doi.org/10.4324/9780203126332

Ochoa, S., Cervantes, G., Jacobo, C., y Flores, J. (2020). Knowledge and innovation in Mexican agricultural organizations. Economies, 8(4), 1-12. doi: https://doi:10.3390/economies8040103

Ojeda, M., Mayer, A., y Solomon, B. (2008). Economic valuation of environmental services sustained by water flows in the Yaqui River Delta. Ecological Economics, 65(1), 155-166. doi: https://doi.org/10.1016/j.agwat.2008.01.016

Olavarría, M. (2000). Dimensión territorial y espacio vivido en los pueblos yaquis. Dimensión Antropológica, 20(7), 79-99. Recuperado de https://www. dimensionantropologica.inah.gob.mx/?p=1001

Olmedo, V. (2017). Determinación de indicadores de gestión en los módulos del distrito de riego No. 041 río Yaqui (Sonora, México) (tesis doctoral). Universidad de Córdoba. Recuperado de https://helvia.uco.es/xmlui/bitstream/ handle $/ 10396 / 16301 / 2018000001739$. pdf?sequence=1\&isAllowed=y

Otero, G. (2004). ¿Adiós al campesinado? Democracia y formación política de las clases en el México rural. México: Universidad Autónoma de Zacatecas, Simon Fraser University y Miguel Ángel Porrúa.

Prats, J. (2003). El concepto y el análisis de la gobernabilidad. Revista Instituciones y Desarrollo (14-15), 239-269. Recuperado de https://www.ses. unam.mx/docencia/2007II/Lecturas/Mod3_Oriol.pdf

Pubill, A., y Buzinde, C. (2021). Indigenous self-representations in the touristic sphere. Annals of Tourism Research (86), 1-13. doi: https://doi.or$\mathrm{g} / 10.1016 / \mathrm{j}$.annals.2020.103099

Ramos, L. (2016). Educación superior y el estudio de realidades locales, una aproximación a la estructuración del campo organizacional en un municipio. Revista Análisis Organizacional (número especial), 504-542.

Raufflet, E. (2017). Responsabilidad social corporativa y desarrollo sustentable: programa de investigación para Latinoamérica. En G. Ramírez y D. Gonzales-Miranda (eds.), Tratado de estudios organizacionales, teorización sobre el campo (pp. 189-200). Medellín: Universidad EAFIT (Escuela de Administración, Finanzas e Instituto Tecnológico), Universidad Autónoma Latinoamericana, Red Mexicana de Estudios Organizacionales y Sage Publications.

Reay, T., y Hinings, C. R. (2005). The recomposition of an organizational field: health care in Alberta. Organization Studies, 26(3), 351-384. doi:https:// doi.org/10.1177/0170840605050872

Rivera-Rodríguez, I. (2016). La cuestión agraria a los cien años de la Constitución Política. En E. Ferrer y L. R. Guerrero (coords.), Derechos del pueblo mexicano. México a través de sus constituciones (pp. 578-585). México: Cámara de Diputados del H. Congreso de la Unión, LXIII Legislatura, Suprema Corte de Justicia de la Nación, Instituto Nacional Electoral, Comisión Nacional de los Derechos Humanos, Senado de la República, LXIII Legislatura, Tribunal Electoral del Poder Judicial de la Federación y Miguel Ángel Porrúa.

Rivera, L. (2018). Formación e institucionalización del campo organizacional de la cinematografía colombiana (tesis de maestría). Universidad Fe- 
deral do Rio Grande do Sul. Recuperado de https://lume.ufrgs.br/handle/10183/184647?locale-attribute=es

Robledo-Silva, P. (2020). ¿Cómo entender el territorio? Anotaciones sobre cómo ampliar el concepto. En J. Rincón y N. Cabezas (eds.), Ordenación del territorio, ciudad y derecho urbano. Competencias, instrumentos de planificación y desafíos (pp. 45-102). Colombia: Universidad Externado de Colombia.

Rosa, A. de la, Pérez, M., Contreras, J., y Hernández, A. (2017). El campo organizacional de la Cooperación Internacional para el Desarrollo: un primer acercamiento. Revista Internacional de Cooperación y Desarrollo, 4(1), 81103. doi: https: / / doi.org /10.21500/23825014.3118

Sánchez, J. (2020). Campo organizacional y cambio institucional en el Hospital Psiquiátrico del Valle entre 1956 y 1970. Summa Psicológica, 17(1), 49-61. doi: https: //doi.org/10.18774/0719-448x.2020.17.419

Schneider, S., y Peyré, I. (2006). Territorio y enfoque territorial: de las referencias cognitivas a los aportes aplicados al análisis de los procesos socio-rurales. En M. Manzanal, G. Neiman, y M. Lattuada (eds.), Desarrollo rural, organizaciones, instituciones y territorio (pp. 71-102). Buenos Aires: Ciccus.

Scott, R. (2012a). Teoría institucional y organizaciones. En R. Vergara (comp.), Organización e instituciones (pp. 163-185). México: Siglo XXI Editores.

Scott, R. (2012b). Teoría contemporánea institucional. En V. Rodolfo (ed.), Organización e instituciones (pp. 186-222). México: Siglo XXI Editores.

Scott, R. (2014). Institutions and Organizations. Idea, Interests and Identities. Los Ángeles: Sage Publications.

Servicio de Información Agroalimentaria y Pesquera (SIAP). (2016). Plano del valle del Yaqui. Colección general. Recuperado de http://w2.siap.sagarpa. gob.mx/mapoteca/

Silva, A. (2016). La influencia del contexto comunitario rural en la conformación de un campo organizacional textil en la zona Puebla-Tlaxcala. Referentes institucionales, historia e identificación (tesis doctoral). Universidad Autónoma Metropolitana. Recuperado de http://tesiuami.izt.uam.mx/uam/ aspuam / presentatesis. php?recno=17692\&docs=UAMI17692.pdf

Sosa, M. (2012). ¿Cómo entender el territorio? Guatemala: Editorial Cara Paren. Tolbert, P., y Zucker, L. (1996). The institutionalization of institutional theory. En S. Clegg, C. Hardy y W. Nord (eds.), Handbook of Organization Studies (pp. 175-190). Londres: Sage Publications.

Trasviña, A., Bórquez, R., Leal, J., Castro, L., y Gutiérrez, M. (2018). Rehabilitación de un suelo salino con yeso agrícola en un cultivo de nogal en el valle del Yaqui. Revista Terra Latinoamericana (36), 85-90. doi: https:// doi. org/10.28940/terra.v36i1.310

Uribe-García, J. (1994). El noroeste: Sonora. En D. Piñera Ramírez (ed.), Visión histórica de la frontera norte de México. De la revolución a la Segunda Guerra Mundial (pp. 211-219). Mexicali: Universidad Autónoma de Baja California.

Vargas-Hernández, J. G. (2008). Perspectivas del institucionalismo y neoinstitucionalismo. Ciencia Administrativa-Ensayos (1), 47-58. Recuperado de https: / / www.uv.mx/iiesca/files/2012/12/perspectivas2008-1.pdf 
Williamson, 0. (2002). The theory of the firm as governance structure: from choice to contract. Journal of Economic Perspectives, 16(3), 171-195. doi: https: / / doi.org/10.1257/089533002760278776 